\title{
Coleção de microalgas de ambientes dulciaquícolas naturais da Bahia, Brasil, como potencial fonte para a produção de biocombustíveis: uma abordagem taxonômica
}

Maria Cristina de Queiroz Mendes ${ }^{1,4,5}$, Augusto Abilio Comas Gonzalez², Mariângela Menezes ${ }^{3}$, José Marcos de Castro Nunes ${ }^{4}$, Solange Pereira ${ }^{1}$ e Iracema Andrade Nascimento ${ }^{1}$

Recebido em 21/06/2011. Aceito em 14/06/2012

\begin{abstract}
RESUMO
(Coleção de microalgas de ambientes dulciaquícolas naturais da Bahia, Brasil, como potencial fonte para a produção de biocombustíveis: uma abordagem taxonômica). O presente trabalho envolveu a identificação taxonômica de espécies nativas de microalgas (isoladas de ecossistemas dulciaquícolas localizados nos arredores de Salvador, Bahia) integrantes da Coleção de Microalgas dulciaquícolas do LABIOMAR/IB/UFBA, visando estudos taxonômicos mais aprofundados (ultraestruturais e moleculares) e experimentos que possam avaliar sua capacidade para suprir cadeias produtivas de biocombustíveis. As coletas foram realizadas nos arredores de Salvador, Bahia, Brasil. A identificação das espécies foi efetuada com base em caracteres morfológicos. Foram identificados 19 táxons, 12 em nível de espécie e nove em nível de gênero, sendo 14 Chlorophyceae (Chlamydomonas sp1, Chlamydomonas sp2, Chlamydomonas sp3, Chlamydocapsa bacillus (Teiling) Fott, Chlorococcum sp1, Chlorococcum sp2), Coelastrum indicum Turn.. Coelastrum microporum Nägeli, Desmodesmus brasiliensis (Bohl.) Hegew, Scenedesmum obliquus (Turpin) Kütz, Ankistrodesmus falcatus (Corda) Ralfs, Ankistrodesmus fusiformis Corda, Kirchneriella lunaris (Kirchner.) Möbius, Pseudokirchneriella subcapitata (Korshikov) F. Hindák), três Trebouxiophyceae (Botryococcus braunii Kütz., Botryococcus terribilis Komárek et Marvan e Chlorella vulgaris Beijerinck), uma Bacillariophyceae (Nitzschia sp.) e uma Cyanobacteria (Synechocystis sp.).
\end{abstract}

Palavras-chave: coleção de microalgas, taxonomia, biodiversidade, biocombustíveis

\begin{abstract}
(Collection of microalgae from natural freshwater environments of Bahia, Brazil, as a potential source for biofuel production: a taxonomic approach). This study identified native species of microalgae (maintained at LABIOMAR/IB/ UFBA Collection of Freshwater Microalgae) to indicate their potential to supply the biofuel production chain. Samples were collected in freshwater ecosystems around Salvador, Bahia, Brazil. Species identification was based in morphological characteristics. Nineteen species were isolated and identified, 12 at the level of species and nine at the level of genus: 14 Chlorophyceae (Chlamydomonas sp1, Chlamydomonas sp2, Chlamydomonas sp3, Chlamydocapsa bacillus (Teiling) Fott, Chlorococcum sp1, Chlorococcum sp2, Coelastrum indicum Turn. Coelastrum microporum Nägeli, Desmodesmus brasiliensis (Bohl.) Hegew, Scenedesmum obliquus (Turpin) Kütz, Ankistrodesmus falcatus (Corda) Ralfs, Ankistrodesmus fusiformis Corda, Kirchneriella lunaris (Kirchner.) Möbius, Pseudokirchneriella subcapitata (Korshikov) F. Hindák), three Trebouxiophyceae (Botryococcus braunii Kütz., Botryococcus terribilis Komárek et Marvan and Chlorella vulgaris Beijerinck), one Bacillariophyceae (Nitzschia sp.) and one Cyanobacteria (Synechocystis sp.).
\end{abstract}

Key words: biofuels, biodiversity, collection microalgae, taxonomy

\footnotetext{
${ }^{1}$ Universidade Federal da Bahia, Instituto de Biologia, Salvador, BA, Brasil

${ }^{2}$ Centro de Estudios Ambientales de Cienfuegos, Cienfuegos, Cuba

${ }^{3}$ Universidade Federal do Rio de Janeiro, Museu Nacional, Departamento de Ficologia, Rio de Janeiro, RJ, Brasil

${ }^{4}$ Universidade do Estado da Bahia, Programa de Pós-Graduação em Biodiversidade Vegetal, Alagoinhas, BA, Brasil

${ }^{5}$ Autor para correspondência: mcqm@uol.com.br
} 


\section{Introdução}

A comunidade científica tem recentemente focado considerável atenção no desenvolvimento de fontes de energia renováveis das mais variáveis fontes biológicas, como alternativa para os combustíveis fosseis, no sentido de minimizar os impactos do aquecimento global (Dismukes et al. 2006; Mutanda et al. 2011).

A seleção de matéria prima tem como base altos rendimentos, pequena duração, baixo custo de produção e menor uso da terra. Nesse contexto as microalgas produtoras de lipídios apresentam potencial para substituir o convencional óleo diesel (Mutanda et al. 2011).

As microalgas representam um grupo de microorganismos extremamente diverso, altamente especializado e de ampla distribuição biogeográfica, com capacidade de seqüestrar $\mathrm{CO}_{2}$ pré-existente através de sua atividade fotossintética e acumular biomassa rápida e eficientemente quando comparada às plantas terrestres (Pienkos \& Darzins 2009)

Dessa forma, as microalgas são uma possibilidade versátil uma vez que, por originarem biocombustíveis de terceira geração (derivados de biomassa), podem dar origem além de biodiesel, a etanol, bioquerosene, bioplásticos, biohidrogênio, biogás (metano) e intermediários químicos para o setor petroquímico (Singh \& Gu 2010).

Em comparação às plantas oleaginosas, as microalgas apresentam maior eficiência em produtividade lipídica (algumas espécies podendo alcançar até $80 \%$ do peso seco em óleo), reproduzem-se rapidamente e durante todo o ano, requerem menor área de cultivo, além de utilizarem áreas não agriculturáveis, requerem menos água, podem ser cultivadas em água não potável, além de serem $\mathrm{CO}_{2}$ neutras e sequestrarem $\mathrm{CO}_{2}$ pré-existente (Singh \& Gu 2010).

Embora constituam fontes energéticas alternativas, existem alguns entraves iniciais para a produção de biomassa microalgal como: (i) identificação de cepas nativas ricas em lipídios (triacilgliceróis, ácidos graxos e hidrocarbonetos), (ii) identificação de cepas de rápido crescimento, concomitante com a produção de reservas lipídicas (iii) identificação de microalgas capazes de resiliência ambiental para adaptação às condições de cultivo.

Neste sentido, a prospecção de cepas locais que atendam às demandas de cultivo é o primeiro passo para o desenvolvimento de matéria-prima algácea efetivamente viável e eco-compatível.

Adicionalmente, a prospecção de espécies nativas com esse potencial é fator preponderante para o conhecimento da biodiversidade local e desenvolvimento sustentável, além de evitar a introdução de espécies oriundas de outras localidades, com riscos de introdução de espécies alóctones. Essa bioprospecção gera inventários de microalgas escassos em determinadas regiões do Brasil, principalmente na região nordeste, além de incrementar a criação de coleções de cultivo de microalgas para a região, suprindo dessa forma a demanda de organismos nos mais variados segmentos da pesquisa científica.

Existe carência de inventários de microalgas dulciaquícolas para o Estado da Bahia. Os estudos efetuados por Zimmermann (1917, 1918 e 1919), com enfoque em diatomáceas e a referência de Senna (1969) apud em Martins et al. (1991), à 12 gêneros, sem identificação em nível específico, são exemplos dessa carência. Merece destaque o trabalho de Martins et al. (1991), que apresenta um estudo qualitativo da flora ficológica no Dique do Tororó, Salvador, Bahia.

Considerando que um dos grandes problemas dos estudos taxonômicos de microalgas está relacionado à dificuldade de se encontrarem formas reprodutivas no ambiente natural, a manutenção de cepas em coleções de cultivo de microalgas fornece maiores subsídios para uma identificação taxonômica mais precisa, não só para estudos do ciclo de vida, às vezes essenciais para uma correta identificação em alguns grupos, assim como para estudos mais aprofundados (ultraestruturais e moleculares).

Durante as décadas passadas, extensivas coleções de cultivo de microalgas foram criadas por pesquisadores em diferentes países para suprir a demanda de pesquisas nos mais variados segmentos (Mata et al. 2010). No Brasil algumas coleções de microalgas encontram-se disponíveis para o uso da comunidade científica (Lourenço 2006), porém restritas, em sua maioria, às regiões sul e sudeste.

Assim, para o preenchimento dessa lacuna no Estado da Bahia, o presente trabalho tem por objetivo apresentar a descrição taxonômica das microalgas nativas, integrantes da Coleção de Cultivo de Microalgas do LABIOMAR/IB/ UFBA, coletadas em ambientes dulciaquícolas da Bahia, disponibilizando-as para estudos taxonômicos mais aprofundados (ultraestruturais e moleculares) e para experimentos que possam avaliar sua capacidade para suprir cadeias produtivas de biocombustíveis.

\section{Material e métodos}

Coleta, isolamento e manutenção das espécies de microalgas seguiram o protocolo de formação da Coleção de Cultivo de Microalgas do Laboratório de Biologia Marinha e Biomonitoramento- LABIOMAR, do Instituto de Biologia da Universidade Federal da Bahia. As coletas foram realizadas nas Lagoas do Caixão (12093'26.38”S - 38³9'09.41”W) e Lagoa do Abaeté (1294'56.02”S $38^{\circ} 21^{\prime} 29.40^{\prime \prime} \mathrm{W}$ ), ambas localizadas no município de Salvador, BA, utilizando sistema desenvolvido no próprio LABIOMAR, com capacidade para reter microalgas menores que $100 \mu \mathrm{m}$. Frações da amostra natural foram enriquecidas com meio de cultura CHU-13 modificado (Dayananda 2006), e LC-Oligo (ABNT-NBR 12648/2004). $\mathrm{O}$ isolamento das espécies foi realizado através de pipetagem, com lavagens sucessivas para obtenção de um cultivo unialgal. As cepas foram mantidas em placas estéreis de 
poliestireno com tampa, contendo poços de fundo chato, e posteriormente transferidas para tubos de ensaio com meio de cultura Chu 13 modificado e LC-Oligo (na dependência do tipo de microalga), e mantidas em câmara de germinação a $25^{\circ} \mathrm{C}$, em regime de $24 \mathrm{~h}\left(114 \mu \mathrm{E} / \mathrm{m}^{2} \cdot \mathrm{s}^{-1}\right)$.

A identificação das espécies foi efetuada com base em suas características morfológicas tendo como referência os trabalhos de Komárek \& Marvan (1992), Comas González (1996), Menezes e Bicudo (2008), Fanés et al. (2009) para clorófitas, Round (1990) para diatomáceas e Komárek \& Anagnostidis (1999) para cianobactérias. As imagens foram capturadas utilizando microscópio NIKON ${ }^{\circledR}$ trinocular OPTIPHOT, com câmera Infinity Lite da Lumera Scientific ${ }^{\circ} \mathrm{e}$ sistema de captura de imagens Image Pro, versão 5.1.1 da Media Cybernetics ${ }^{\oplus}$, (LABIOMAR, Instituto de Biologia, UFBA), além de microscópio Olympus ${ }^{\circledast}$ BX-51, com sistema de captura de imagens Image Pró-Plus 6.2 (Serviço de Microscopia Eletrônica (SME) - Centro de Pesquisas Gonçalo Moniz - FIOCRUZ).

\section{Resultados e discussão}

Em cumprimento à primeira etapa da prospecção de microalgas (coleta, isolamento, identificação e manutenção no Banco de Microalgas), obteve-se o isolamento de 19 táxons, 12 em nível de espécie e sete em nível de gênero, sendo 14 da classe Chlorophyceae (Chlamydomonas sp1, Chlamydomonas sp2, Chlamydomonas sp3, Chlorococcum sp1, Chlorococcum sp2, Chlamydocapsa bacillus, Coelastrum indicum, Coelastrum microporum, Desmodesmus brasiliensis, Scenedesmus obliquus, Ankistrodesmus falcatum, Askistrodesmus fusiformis, Kirchneriella lunaris, Pseudokirchneriella subcapitata), três da classe Trebouxiophyceae (Botryococcus braunii, Botryococcus terribilis e Chlorella vulgaris), uma da classe Bacillariophyceae (Nitzschia sp.) e uma da classe Cyanophyceae (Synechocystis sp.), com descrição e classificação taxonômica apresentadas a seguir:

\section{Classe Chlorophyceae}

Família Chlamydomonadaceae

Chlamydomonas sp1 (Fig. 1)

Células ovóides 7,9-10,5 x 5,8-9,5 $\mu$ m. Cloroplasto único poculiforme, com pirenóide. Estigma anterior. Dois flagelos apicais iguais. Após a perda dos flagelos, células envoltas por mucilagem. Reprodução assexuada e por conjugação. Chlamydomonas sp2 (Fig. 2)

Células arredondadas, 7,1-7,7 $\mu \mathrm{m}$. Cloroplasto único, poculiforme, com pirenóide. Estigma localizado anteriormente. Dois flagelos apicais.

Chlamydomonas sp3 (Fig. 3)

Células elipsóides, 7,45-8,57 x 4,28-7,71 $\mu \mathrm{m}$. Cloroplasto único, lateral, com pirenóide na região mediana. Estigma anterior. Dois flagelos apicais iguais.
Família Chlorococcaceae

Chlorococcum sp1 (Fig. 4)

Células esféricas, solitárias ou formando grupos, sem mucilagem, 6,0-16,5 $\mu \mathrm{m}$ de diâmetro. Cloroplasto poculiforme. Com o aumento do material genético ocorre aumento no tamanho e o cloroplasto assume a forma estrelar. Pirenóide com capa de amido descontínua. Vacúolos contráteis. Reprodução por zoósporos elipsoidais, tipo Chlamydomonas, 5,5-6,0 x 2,5-4,0 $\mu \mathrm{m}$.

Chlorococcum sp2 (Fig. 5)

Células esféricas, solitárias ou formando grupos, 8,0$18,75 \mu \mathrm{m}$ de diâmetro. Cloroplasto poculiforme com um pirenóide. Reprodução por zoósporos elipsoidais, tipo Chlamydomonas, 2,7-4,5 x 1,5-1,7 $\mu \mathrm{m}$.

Família Palmellopsidaceae

Chlamydocapsa bacillus (Teiling) Fott (Fig. 6)

Colônias mucilaginosas com grupos de 2-16 células, mucilagem estratificada envolvendo cada célula e os grupos de células. Células elípticas, pólos arredondados, 9,0-12,0 x 4,0$7,0 \mu \mathrm{m}$. Cloroplasto único, perfurado, às vezes fragmentado em unidades discóides, com um pirenóide aproximadamente lateral. Estigma anterior, elíptico. Dois vacúolos contráteis. Reprodução por 2-4-8 zoósporos dentro da célula mãe. Em culturas mais velhas, a mucilagem torna-se mais lamelada. Primeira citação para a Bahia.

Família Scenedesmaceae Coelastrum indicum Turn. (Fig. 7)

Cenóbios esféricos com 8-32 células. Células poliédricas em vista polar, 6,3 - 14,3 $\mu \mathrm{m}$, com apêndices de união cilíndricos e curtos. Cloroplasto único parietal, com pirenóide. Primeira citação para a Bahia.

Coelastrum microporum Nägeli (Fig. 8)

Cenóbios esféricos, com células unidas diretamente por suas paredes, Células esféricas ou levemente ovais, pólos cônicos arredondados, 8,5-11,1 $\mu \mathrm{m}$ diâmetro. Cloroplasto único, parietal, com pirenóide.

Desmodesmus brasiliensis (Bohl.) Hegew (Fig. 9)

Cenóbios com quatro células alinhadas linearmente. Células elípticas, pólos arredondados com 1-3 dentículos, 6,4-10,3 x 3,3-5,0 $\mu \mathrm{m}$. Parede celular com costelas longitudinais às vezes descontinuas. Cloroplasto único, parietal com pirenóide. Scenedesmus obliquus (Turpin) Kützing (Fig. 10)

Cenóbios com 2-4-8 células alinhadas linearmente ou irregularmente alternadas. Células fusiformes, extremidades pontiagudas, células internas ligeiramente retas, células externas curvas, 9,4-13,4 x 3,4-6,7 $\mu \mathrm{m}$. Cloroplasto único, parietal, com pirenóide.

Primeira citação para a Bahia.

Família Selenastraceae

Ankistrodesmus falcatus (Corda) Ralfs (Fig. 11)

Colônias com células dispostas em fascículos. Células fusiformes, ligeira e irregularmente curvas com extremidades 



Figuras 1-7. Imagens em microscopia óptica de espécies de microalgas nativas isoladas e identificadas, integrantes da Coleção de Culturas de Microalgas do LABIOMAR/IB/UFBA. 1. Chlamydomonas sp. 1: detalhe reprodução por conjugação; 2. Chlamydomonas sp. 2; 3. Chlamydomonas sp. 3; 4. Chlorococcum sp1:. 4A: células vegetativas; $4 \mathrm{~B}$ : detalhe eclosão dos zoósporos; 5 . Chlorococcum sp2: detalhe células vegetativas e zoósporos; 6. Chlamydocapsa bacillus (Teiling) Fott; 7. Coelastrum indicum Turn. Barra: $10 \mu \mathrm{m}$.

atenuadas, 33,8-51,4 x 1,4-2,9 $\mu \mathrm{m}$. Cloroplasto único, parietal. Primeira citação para a Bahia.

Ankistrodesmus fusiformis Corda (Fig. 12)

Colônias com células dispostas radialmente ou cruciada. Células fusiformes, ligeiramente cilíndricas em sua porção media, mais ou menos retas ou irregularmente curvas, extremidades atenuadas gradualmente, $37,8-56,0$ x 2,8-5,0 $\mu \mathrm{m}$. Cloroplasto único, parietal.

Primeira citação para a Bahia.

Kirchneriella lunaris (Kirchner.) Möbius (Fig. 13)

Colônias com 2-10 células, distribuídas irregularmente em matriz mucilaginosa inconspícua. Células lunadas, extremidades atenuadas e pontiagudas, 3,1-5,6 $\mu \mathrm{m}$ de contorno celular. Cloroplasto único, parietal.

Primeira citação para a Bahia.

Pseudokirchneriella subcapitata (Korshikov) F. Hindák (Fig 14)
Colônia com 2-32 células embebidas em mucilagem não estruturada. Células lunadas a fusiformis, extremidades pontiagudas ou arredondadas $1,4-2,8 \mu \mathrm{m}$ de largura $\times 7,1-10,7$ $\mu \mathrm{m}$ de abertura entre as extremidades. Cloroplasto único e parietal. Reprodução por autósporos. Ausência de pirenóide. Primeira citação para a Bahia.

Classe Trebouxiophyceae

\section{Família Botryococcaceae}

Botryococcus braunii Kütz. (Fig. 15)

Colônias densas, 125,0-155,0 $\mu \mathrm{m}$ em seu maior diâmetro, formada por subcolônias, 52,5-80,0 $\mu \mathrm{m}$ de diâmetro, interligadas por cordões mucilaginosos. Células obovóides, 11,2-15,0 x 7,5-12,5 $\mu \mathrm{m}$, emergindo da colônia em até $1 / 3$ de seu comprimento. Presença de capacete mucilaginoso hialino sobre as porções apicais em algumas células. Cloroplasto único parietal. Reprodução por 2 autósporos alongados. Primeira citação para a Bahia.

Botryococcus terribilis Komárek et Marvan (Fig.16)

Colônias arredondadas, irregulares, formada por subcolônias, 35,7-157,0 $\mu \mathrm{m}$ diâmetro, interligadas por cordões mucilaginosos, com células densamente arranjadas dispostas radialmente na periferia da mucilagem. Mucilagem colonial com projeções irregulares. Células alongadas, obovadas, 8,0-10,0 x 5,0-9,0 $\mu \mathrm{m}$, ápices totalmente recobertos pela mucilagem. Cloroplasto único, parietal. Reprodução por 2(4) autósporos. Primeira citação para a Bahia.

Família Chlorellaceae

Chlorella vulgaris Beijerinck (Fig. 17)

Células jovens elipsóides e as adultas, esféricas, 2,3-4,0 x 2,4-2,8 $\mu \mathrm{m}$, isoladas ou em pequenos aglomerados transitórios. Cloroplasto único, parietal ocupando $3 / 4$ ou $1 \frac{1}{2}$ da célula, presença de pirenóide. Reprodução por 2-4-8 autósporos. Autósporos elípticos.

Primeira citação para a Bahia.

Classe Bacillariophyceae

Família Bacillariaceae

Nitzschia sp. (Fig. 18)

Vista valvar linear com ápices arredondados. Dois plastídios dispostos simetricamente um em cada lado do plano medial transapical. Eixo apical com 16,3-19,7 $\mu \mathrm{m}$. Eixo transapical 3,4-3,7 $\mu \mathrm{m}$.

Primeira citação para a Bahia.

Classe Cyanophyceae (Cyanobacteria)

Familia Merismopediaceae

Synechocystis sp. (Fig. 19)

Células globosas ou ovais, com ligeiro movimento, solitárias ou em dupla, com um pálido conteúdo verde azulado. 1,4-3,1 $\mu \mathrm{m}$ de diâmetro.

Primeira citação para a Bahia. 


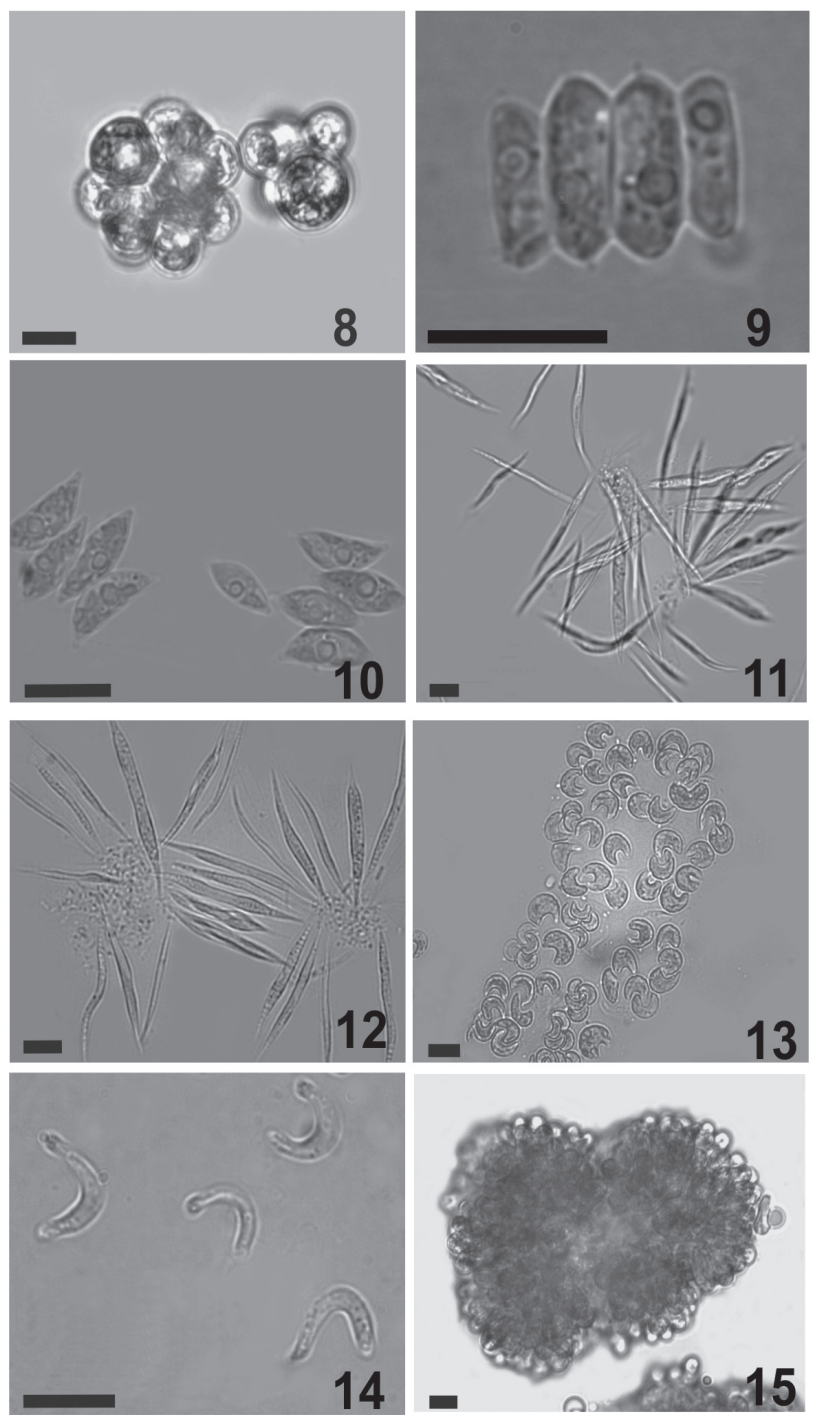

Figuras 8-15. Imagens em microscopia óptica de espécies de microalgas nativas isoladas e identificadas, integrantes da Coleção de Culturas de Microalgas do LABIOMAR/IB/UFBA: Fig. 8. Coelastrum microporum Nägeli; 9. Desmodesmus brasiliensis (Bohl.) Hegew; 10. Scenedesmus obliquus (Turpin) Kützing; 11 Ankistrodesmus falcatus (Corda) Ralfs; 12. Ankistrodesmus fusiformis Corda; 13. Kirchneriella lunaris (Kirchner.) Möbius; 14. Pseudokirchneriella subcapitata (Korshikov) F. Hindák. Barra: $10 \mu \mathrm{m}$; 15. Botryococcus braunii Kütz.

A má distribuição das coleções de cultivo de microalgas no Brasil foi bem caracterizada por Lourenço (2006). Das 44 coleções existentes, apenas oito (18\%) estão localizadas na região nordeste, enquanto as regiões sul e sudeste concentram a maioria (76\%), com 33 coleções de cultura de microalgas dulciaquícolas/marinhas/estuarinas. Dessa forma, torna-se evidente a necessidade da ampliação dessas coleções, não só na região nordeste, como nas demais regiões carentes (norte e centro-oeste).

Este trabalho além de ampliar o conhecimento da biodiversidade local, se traduz numa ferramenta auxiliar para identificação de microalgas aos mais variados segmentos (estudantes de graduação e pós-graduação, relatórios técnicos), através das imagens disponibilizadas.
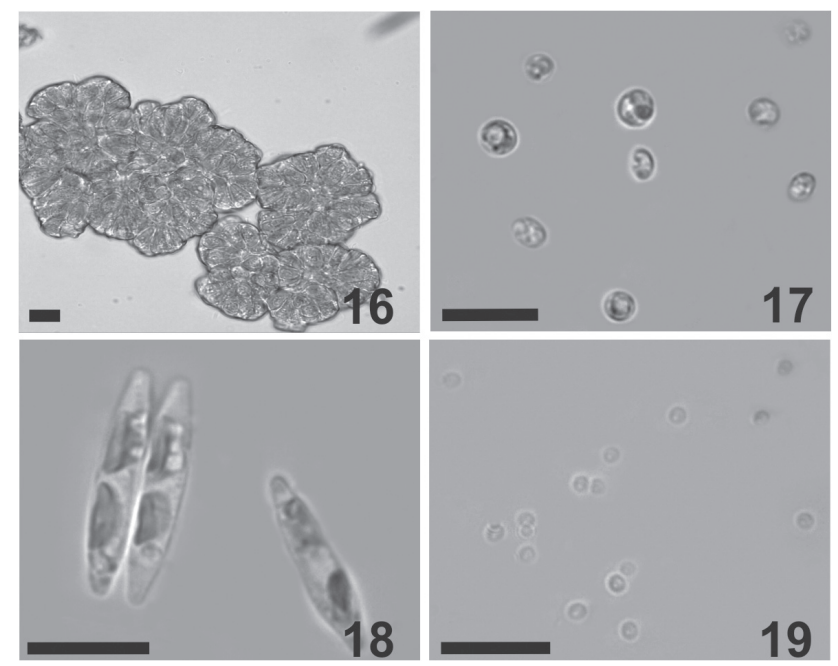

Figuras 16-19. Imagens em microscopia óptica de espécies de microalgas nativas isoladas e identificadas, integrantes da Coleção de Culturas de Microalgas do LABIOMAR/IB/UFBA. 16. Botryococcus terribilis Komárek et Marvan; 17. Chlorella vulgaris Beijerinck; 18. Nitzschia sp.; 19. Synechocystis sp. Barra: $10 \mu \mathrm{m}$.

\section{Agradecimentos}

Ao Conselho Nacional de Desenvolvimento Científico e Tecnológico (CNPq), pelo apoio à pesquisa e concessão de bolsas - Processo 574712/2008-9 e ao Serviço de Microscopia Eletrônica do Centro de Pesquisa Gonçalo Muniz Fundação Oswaldo Cruz (FIOCRUZ), pela disponibilização do sistema de captura de imagens ópticas.

\section{Referências bibliográficas}

ABNT - Associação Brasileira de Normas Técnicas. 2004. Ecotoxicologia aquática - Toxicidade crônica - Método de ensaio com algas (Chlorophyceae). Pp.1-20. Rio de Janeiro, Projeto NBR 12648:2004. $28^{\mathrm{a}}$ reunião.

Comas González, A. 1996. Las Chlorococcales dulciacuícolas de Cuba. Berlin, Stuttgart, Gebrüder Borntraeger Verlagsbuchhandlung.

Daynanda, C.; Sarada, R.; Rani, M.U.; Shamala, T.R. \& Ravishankar, G.A., 2006. Autotrophic cultivation of B. braunii for the product. of hydrocarbons and exopolysaccharides in various media. Biomass \& Bioenergy 31: 87-93.

Fanés. I.; Sánchez, P. \& Comas, A. 2009: Contribution to the taxonomic study of the family Botryococcaceae (Trebouxiophyceae, Chlorophyta) in southern Spain. Cryptogamie Algologie 30(1): 17-30.

Komarék, J. \& Marvan, P., 1992.Morphological Diff. in Natural Populations of the Genus Botryococcus (Chlorophyceae). Archiv fuer Protistenkunde 141: 65-100.

Komárek, J. \& Anagnostidis, K. 1999. Cyanoprokaryota. 1. Tell Chroococcales. In: Ettl, H.; Gardner, G.; Heynig, H.\& Mollenheuer, D. (Eds.). Süsswasserflora von Mittleleuropa. Jena, Gustav Fischer.

Lourenço, S.O. 2006. Cultivo de Microalgas Marinhas: Princípios e Aplicações. São Carlos, Rima Editora.

Martins, D.V., Sant'Anna, C.L. \& Oliveira, O.C. 1991. Estudo qualitativo do fitoplâncton do Dique do Tororó, Salvador, Bahia, Brasil. Revista Brasileira de Biologia 51(2): 445-453.

Mata, T.M.; Martins, A.A. \& Caeano, N.S. 2010. Microalgae for biodiesel production and other applications: a review. Renewable \& Sustainable Energy Reviews 14: 217-232. 
Menezes, M \& Bicudo, C.E., (2008). Flagellate green algae from four water bodies in the state of Rio de Janeiro, Southeast Brazil. Hoehnea 35(3): 435-468.

Round, F.E.; Crawford, R.M. \& Mann, D.G. 1990. The Diatoms, Biology \& Morphology of the Genera. Cambridge, Cambridge University Press.

Pienkos, P.T. \& Darzins A. 2009. The promisse and challengers of microalgal-derived biofuels. Biofuels, Bioproducts \& Biorefining 3: 431-440.

Singh, J., \& Gu, S. 2010. Commercialization potential of microalgae for biofuels production. Renewable and Sustainable Energy Reviews 14(9): 2596-2610.

Zimmermann, C., 1917. VI. Contribuição para o estudo das diatomáceas dos Estados Unidos do Brasil, Broteria 15: 30-45.

Zimmermann, C., 1918. VII. Contribuição para o estudo das diatomáceas dos Estados Unidos do Brasil, Broteria 16: 8-24.

Zimmermann, C., 1919. IX. Contribuição para o estudo das diatomáceas dos Estados Unidos do Brasil, Broteria 17: 5-16. 